\title{
Dietrich Briesemeister
}

(Jena)

\section{AMERIgo VESPUCCIS BRIEFE ÜBER DIE NEUE WELT}

Kaum ein anderes Textkorpus hat zu Beginn der Neuzeit das Bild von der Neuen Welt in Europa so nachhaltig geprägt wie die unter Vespuccis Namen in zahlreichen lateinischen und volkssprachlichen Ausgaben und illustrierten Flugschriften verbreiteten Berichte über die Entdeckung des bald danach Brasilien genannten Teils von Amerika. Der vierte Kontinent trägt auch nicht den Namen des Kolumbus, sondern in einem typisch humanistischen Wortspiel schlug Matthias Ringmann Philesius (1482-1511) Vespuccis Vornamen zur Benennung des neuen Erdteils vor, die durch Martin Waldseemüllers Cosmographiae introductio (Saint-Dié 1507), den Erdglobus und Waldseemüllers monumentale Weltkarte mit der Bezeichnung »America« (zunächst allerdings nur für den Südteil) in die geographische Literatur und Toponymik einging. Der Cosmographia sind erstmals die Quattuor Navigationes des Vespucci mit der Widmung an Herzog René II. von Lothringen beigedruckt. Ringmann verbindet Wort und Sache im überlieferten Sinn der »Etymologie als Denkform« (Ernst Robert Curtius), wenn er Amerigo als sprechenden Namen deutet: Land (griechisch $\gamma \hat{\eta}=$ Erde, Gebiet) des Americus:

Alia quarta pars per Americum Vesputium [...] inuenta est quam non video cur quis iure vetet $a b$ Americo inuentore sagacis ingenii viro Amerigen quasi Americi terram siue Americam dicendam, cum et Europa et Asia a mulieribus sua sortita sint nomina. ${ }^{1}$

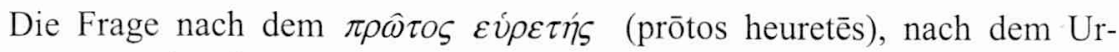
sprung und Erfinder, hatte bereits für das griechische historische Denken eine besondere Bedeutung: Große Erfinder sind nicht nur eine dauernde Ehre für ihren Herkunftsort, sie bilden auch eine eigene Kategorie der "Weisen«. Spezielle Erfinderkataloge finden sich bei Plutarch und Xeno-

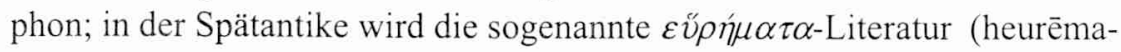
ta) immer umfangreicher. Erst die Renaissance brachte jedoch den Erfin-

1 Cosmographiae introductio, fol. $207 \mathrm{v}^{\circ}$. 
dertopos wieder zur Geltung: Polidoro Virgilio (1470-1555) stellte dafür die umfangreichste Kompilation De inventoribus rerum bereit (spanische Fassung Antwerpen 1550). Mindestens sechzig Drucke, die verlorenen nicht eingerechnet, erschienen allein im ersten Drittel des 16. Jahrhunderts. Unter den frühen siebenunddreißig Ausgaben - in italienischer, französischer, niederländischer, aber bezeichnenderweise weder portugiesischer noch spanischer Sprache - befinden sich allein siebzehn deutsche, denen nur noch dreiundzwanzig lateinische Ausgaben gegenüberstehen. In der umfangreichen und kontroversen Literatur über das frühe Amerika-Bild werden die gedruckten Texte zumeist ohne Bedenken so gelesen, als seien sie von Vespucci selbst verfasst. Dem ist freilich keineswegs so. Nach neueren Forschungen sind lediglich zwei der vier Reisen Vespuccis gesichert. Die massenhafte Verbreitung und der sensationelle Gehalt der Berichte Von der neuw gefunden Region die wol ain welt genent mag werden (1505), von Vil Insulen unnd ein Nüwe welt von wilden nackenden Leuten vormal unbekant (1509), vom Mundus Novus (1504) - um nur einige Titel und Schlagwörter anzuführen - ließen Vespucci als epochemachende Gestalt erscheinen. Es ist hier nicht der Platz, um den verwickelten Vorgang der Textentstehung und -tradierung zu untersuchen, bei der sich verkürzende Exzerpierung und rhetorische amplificatio, anthologische Kompilation von Quellen und Nachrichtenklitterung, Übersetzungen (auch aus zweiter Hand) und Rückübersetzungen, die neue, auf Erfahrung gegründete Wissenschaft und überkommenes Autoritätswissen, Missverständnisse und Macht der Mythen, Sensationsgier und Gewinnspekulation vielfältig überlagern und verquicken. Die überseeische Expansion der Portugiesen und Spanier bot schon früh Stoff für science fiction, für die volkstümliche und fiktionale Aufbereitung im erfundenen Entdeckerbericht, wie etwa die aus dem Italienischen übersetzte Schiffung mit dem Lanndt der Gulden Insel gefunden durch Hern Johan von Angliara Hawptmann des Cristenlichen Künigs von Hispania gar hübsch ding zu hören mit allen yren Leben und sitten (Augsburg 1520) zeigt. In Thomas Morus' Utopia (1515) ist der (fiktive) Erzähler der Weltreisende Hythlodaeus, angeblich ein Portugiese und Begleiter

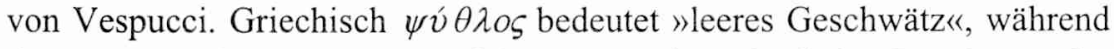
der Vorname des Plauderers auf den Erzengel Raphael, den Beschützer derer, die unterwegs, auf Reisen sind, anspielt. Auch die Wechselwirkungen von ausschmückenden Holzschnittillustrationen, von Bildvorstellungen, auf die Textgestaltung und deren Wandlungen dürften nicht gering veran- 
schlagt werden. Vespucci (1451-1512) selbst hat allerdings auf die postumen Ausgaben und deren geschickte, auf Effekte bedachte Bild- und Textmontagen keinen Einfluss nehmen können. Die Breitenwirkung der Drucke war natürlich ungleich größer als die der heute für authentisch gehaltenen handschriftlichen Briefe, die im Folgenden betrachtet werden sollen. In irgendeiner Form - Abschrift, Auszug, Übersetzung oder unter Hinzufügung anderer mündlicher bzw. schriftlicher Informationen - haben sie den Druckerzeugnissen zugrunde gelegen. Das Verhältnis zwischen Vespuccis Briefen und den Druckfassungen mit ihren Paratexten kann hier nicht näher geprüft werden. Der Vergleich böte reichliche Paradebeispiele für Genettes theoretische Klassifikationen. Es geht, mit Stephen Greenblatt gesprochen, auch nicht in erster Linie darum, zwischen »wahren « und »falschen«, das heißt fiktiven und erfundenen Darstellungen der Neuen Welt, zwischen dem apokryphen und dem authentischen Vespucci zu unterscheiden, sondern um den Versuch, die Repräsentationspraktiken des Florentiners, einige seiner rhetorischen Grundmuster und Redestrategien bei der Inszenierung und Wiedergabe dessen, was er sah, erlebte und tat, hervorzuheben.

Vespuccis Schilderungen und Sprachkunst werden oft überschwänglich gerühmt ob ihrer »pensive seriousness in the face of the unfamiliar«, ihrer »questioning humility « - im Gegensatz zu den »triumphalistischen Aussagen und Erfolgsekstasen« eines Kolumbus, wie Antonello Gerbi meint, der sogar »no conventional idealizations « bei Vespucci feststellen kann. ${ }^{2}$ Auch Eberhard Schmitt bewundert bei Vespucci »eine durch Liebe zum Detail ausgezeichnete, überaus einfühlsame und lebendige Schilderung der angetroffenen Gegebenheiten, insbesondere der Eingeborenenkultur « ${ }^{3}$. Gegenüber solchen Einschätzungen ist jedoch Vorsicht geboten, nicht nur wegen der Authentizitätsproblematik an sich, sondern wegen der hochgradig rhetorischen Gestaltung und des Stilwillens dieser Texte, die unberücksichtigt bleiben. Frauke Gewecke spricht bei ihrer Bewertung der Sprach- und Beschreibungskunst Vespuccis immerhin von »einer gewissen, möglicherweise sogar beabsichtigten Verzerrung « ${ }^{4}$, also von Entstellung, Verfälschung oder einem täuschenden Missverhältnis im Vergleich mit der Wirklichkeit. Verzerrung dürfte allerdings hier nicht der geeignete Ausdruck sein für die

2 Antonello Gerbi (1976: 39-40).

3 Eberhard Schmitt (1984: 170).

4 Frauke Gewecke (1992: 99). 
überhöhende rhetorische Technik und sprachlichen Kunstgriffe, die natürlich die Briefberichte nicht als unbedingt objektive Wiedergabe von Beobachtungen und uneingeschränkt zuverlässige Dokumente erscheinen lassen. Obwohl sich Vespucci wiederholt starker Beglaubigungsformeln bedient und Zeugen aufruft, um Glaubwürdigkeit und Wahrheit seiner Aussagen zu bekräftigen, bleibt der Fiktionalitätsverdacht auch nicht immer ausgeschlossen. Todorov erkennt »eine gewisse rhetorische Bildung « sowie einen starken Leserbezug im Mundus Novus-Text. Im Unterschied zu Kolumbus, der amtliche Schreiben ausfertigte, etwa den Brief an Santángel, habe Vespucci »Literatur« geschrieben, seine privaten Berichte seien geradezu ein »Triumph der Literatur«. In der Tat, nur war Vespucci nicht ihr Autor, sondern unbekannte Hände haben seine Textvorgabe oder andere Vorlagen kunstvoll arrangiert, aufbereitet und unter Verwendung des bekannten Namens ausgestaltet. Dass sich Vespucci seinerseits durchaus auch auf die rhetorische Kunst verstand, soll nun näher betrachtet werden. Der Sohn eines Florentiner Notars war von seinem Onkel, einem Dominikaner, mit der klassischen Bildung vertraut gemacht worden. Seine Äußerung, er wage es nicht, ohne Hilfe eines Lehrers lateinisch zu schreiben, dürfte weniger eine der üblichen Bescheidenheitsfloskeln sein als vielmehr Ausdruck der bekannten Abneigung des Juristenstandes gegenüber den studia humanitatis (oder umgekehrt der Humanisten gegenüber den Advokaten). Er übte sich, betont Vespucci, lediglich pflichtgemäß im Abschreiben lateinischer Grammatik und klassischer Musterautoren. »Vernacula vero lingua nonnihil erubesco. $\ll^{5}$ 1478-1480 begleitete Vespucci als Sekretär eine Gesandtschaft der Signoria in Frankreich und trat anschließend in den Dienst der Medici, als deren Vertreter er seit 1492 in einem Bankhaus in Sevilla arbeitete. In dieser Stellung war er u. a. mit der Finanzierung der Ausrüstung von Schiffen für Kolumbus befasst. Vespuccis Teilnahme an der Expedition unter Führung von Alonso de Hojeda in den Raum von der Amazonasmündung bis in den Golf von Maracaibo 1499 gilt als gesichert. Nach der Rückkunft im April 1500 nach Sevilla berief ihn König Manuel I., der Glückliche, nach Portugal. Vespucci nahm 1501 an der ersten portugiesischen Erkundungsfahrt entlang der brasilianischen Ostküste teil, nachdem Pedro Álvares Cabral im April 1500 dorthin verschlagen wurde und das

5 Zitiert bei Leonardo Olschki (1922: Bd. 2, 124); siehe auch die Würdigung des wissenschaftlichen Briefes in der italienischen Renaissance, ibid., pp. 323-329. 
Land des heiligen Kreuzes, das spätere Brasilien, wahrscheinlich eher zufällig entdeckt hatte. Vespucci war auf der Expedition mit Vermessungsaufgaben und der wissenschaftlichen Beratung betraut. Danach wechselte Vespucci wiederum in kastilische Dienste und stieg 1508 zum Piloto Mayor der Casa de Contratación in Sevilla auf, der Seefahrtsbehörde der Katholischen Könige, dem die Aufsicht über den Handel der Krone in Übersee und die Archivierung der streng geheimen Seekarten, Logbücher und Seefahrtsberichte sowie die Schulung von Steuerleuten oblag, ein politisch also wichtiger Vertrauensposten, der für Vespuccis Ehrgeiz, Ansehen und Wissen als Kosmograph, Nautiker und Finanzberater spricht. Zwei der beiden anderen nach 1507 wiederholt abgedruckten und übersetzten Berichte in den Quattuor Navigationes beruhen jedenfalls nicht auf Vespuccis eigenen Unternehmungen.

Mit seinen Briefen ${ }^{6}$ reiht sich Vespucci in die seit Petrarca reich entfaltete italienische Briefkultur ein. Sie stehen außerdem im Zusammenhang mit der regen diplomatischen Korrespondenz, die schon in der frühen Zeit der atlantischen Entdeckungen zwischen der Iberischen Halbinsel und Italien - insbesondere Florenz und Venedig - aus handelspolitischem Interesse geführt wurde. Pietro Pasquaglio, Botschafter Venedigs am portugiesischen Hof, hielt am 20. August 1501 eine lateinische Oratio vor König Manuel, die kurz vor Weihnachten in Venedig im Druck erschien und in der Brasilien erstmals - wenngleich nicht mit dieser Bezeichnung - erwähnt wird.? Vespucci war sowohl mit den Gebräuchen des italienischen Kanzlei- und Geschäftswesens als auch mit den Briefgepflogenheiten seiner humanistisch gebildeten Landsleute vertraut. Seit der Antike gelten für den Brief in Aufbau und Stil die Gesetze der Rhetorik. Lateinischer Stil wurde in der Schule am Beispiel von Briefen eingeübt. Brief und Rede (oratio) stimmten in der Großgliederung des Aufbaus überein: salutatio, captatio benevolentiae, narratio, petitio, conclusio. Der Brief wird als »Hälfte eines Gespräches « oder, mit den Worten des Erasmus von Rotterdam, als »absentium amicorum quasi mutuus sermo« - als Zwiegespräch und Gedankenaus-

6 Aus den Briefen Vespuccis wird nach folgender Ausgabe zitiert: Mario Pozzi (Hrsg.) (1991): Scopritori e viaggiatori del Cinquecento e del Seicento, Mailand / Neapel: Ricciardi, Bd. 1; die Seitenangaben im Text beziehen sich auf diese Ausgabe.

7 Der Florentiner Bartolomeo Marchioni meldete bereits im Juli 1501 die Entdeckung eines »neuen Landes« - Brasiliens - in seine Heimatstadt, cf. Johannes Pögl (1986: 171-176); siehe auch Luís de Matos (1992: 174-176 [Pietro Pasquaglio]) 
tausch unter räumlich voneinander entfernten Freunden - bestimmt. Er weist deutlich autobiographische Bezüge auf, die bei Vespucci durch die Entdeckungsfahrten, Aufträge und seinen Forscherdrang geprägt werden, so dass der Brief auch der Selbstdarstellung dient, etwa wenn Vespucci darauf hinweist, der häufige Verzicht auf Schlaf wegen der nächtlichen Himmelsbeobachtungen habe ihn mindestens um zehn Jahre seines Lebens gebracht $(252)^{8}$. Die an Lorenzo di Pierfrancesco de' Medici gerichteten Briefe Vespuccis zielen auf eine bestimmte Wirkung und müssen sich daher im Stil an den Rang des Empfängers anpassen: Sie informieren, vermitteln neue Erkenntnisse, geben Erfahrungen weiter, belehren und verfolgen Interessen, wollen aber auch unterhalten (Lektüre »come frutta dipoi levata la mensa« [225], als Leckerbissen zum Nachtisch). Vespucci bezeichnet sie als »scritta, menzione, lettera« oder »buona e vera relazione« (261) zum Zweck von »dare notizia« oder »dare conto per lettera«.

Die Briefgattung erlangt im Zeitalter der Entdeckungen eine außerordentliche Bedeutung. Der Brief gibt als offizieller Bericht eine urkundliche Bestätigung für die Inbesitznahme, als Rechenschaftsbericht bestätigt er die Ausführung eines Auftrags (bei Kolumbus, Hernán Cortés). Der Brief dient der Übermittlung und aktuellen Kommentierung von Neuigkeiten (bei Petrus Martyr de Anghiera), als polemische öffentliche Anklagerede (bei Las Casas). Der gelehrte Brief rückt in die Nähe des Essays oder Traktats (Lehrbrief). Der dokumentarische Quellenwert von Briefen muss doch behutsam entschlüsselt werden. In dem 1502 aus Lissabon unmittelbar nach der Rückkehr an den Mediceer gerichteten Brief betont Vespucci, dass er vom portugiesischen König mit dem Auftrag (»comesione« [279]) ausgesandt war, um »cercare nuove terre (Neuland, nicht Inseln), um Entdeckungen von bislang Unbekanntem zu machen (»discoprir molto più « [261]), und nicht bloß auf bereits erkundeten Routen zu segeln wie Vasco da Gama (»andar per il discoperto« [245]). Er beginnt die narratio mit einer »dichiarazione« (275) oder descriptio, Beschreibung des Landes (Brasilien), der Tier- und Pflanzenwelt sowie der Menschen und ihrer mores nach der Art der antiken Buntschriftstellerei, des Länderlobs und der Darstellung der Natur. Dieser Briefteil bildet schon für sich ein virtuoses Stück rhetorischer Schreibkunst, mit dem Vespucci dem hochgestellten Briefempfänger als Vorausbericht (»tutto sotto brevità e solo capita rerum delle cose" 
[280]) und »Auszug« aus Aufzeichnungen - vielleicht dem Tagebuch? eine Probe seiner Reiseerfahrung gibt, um ihn erwartungsvoll einzustimmen auf das nach der Rückkehr in gelehrter Muße und möglichst mit Förderung des Mediceers »tutto a minuto « auszuarbeitende und ihm dann auch zu widmende Werk. Es soll zugleich und vor allem Vespuccis eigenen Ruhm für die Nachwelt sichern (»per lasciare di me dopo la morte qualque fama« [274], oder an anderer Stelle: »Spero venir in fama lungo secolo« [252]). Er rühmt sich, seinem Magnifico Padron nicht nur aus fernen Ländern, sondern - was nur wenigen gegeben ist - sogar von hoher See aus schreiben zu können (»scrivere non solo di lunga terra, ma de lo alto mare« [249]). Vespucci beansprucht selbstbewusst fama für seine Entdeckungen, wie zuvor Poeten, Philologen, Feldherrn oder Herrscher für ihre Werke und Taten. Die Bezeichnung des neuen Kontinents bestätigt und verewigt seinen Nachruhm.

Die sprachliche, begriffliche Umsetzung seiner Wahrnehmung der Natur dieser in Europa bisher unbekannten Welt ist ihm nur möglich mit den Mitteln eines schon seit der Antike geläufigen Vorrats an Gesichtspunkten und Sprachmustern für eine Natur- und Landschaftsbeschreibung. Es ist das Schema einer ideal gedachten, komponierten, das heißt aus Versatzstücken kunstvoll gefügten Natur als locus amoenus, mit allen seinen stypischen`, schönen Bestandteilen und Merkmalen: Bäumen, Wasserreichtum, lauen Winden, gemäßigtem Klima, üppiger, farbiger Vielfalt an Früchten, Blumen und Getier, Wohlgerüchen und Wohllaut. Alle Sinnesempfindungen werden aufgenommen. ${ }^{9}$ Vespucci kommt es nicht darauf an, spontan seine persönlichen Anmutungen und Beobachtungen auszudrücken, sondern er fasst diese in das vorgeordnete, dem gebildeten Italiener verfügbare, vertraute und verständliche Schaubild. Im Briefbericht hat diese Art und Weise kunstvoller Evozierung durchaus eine kommunikative Funktion, sie ist keineswegs unbrauchbar oder überflüssig, aber sie ist eben auch nicht auf die spezifische, realistische Abbildung der Neuen Welt ausgelegt, sondern spricht die Imagination, die Bilderfindung als Erkenntnisweise an. Bereits das erste Stichwort »terra [...] molto amena« verweist auf die konventionelle Beschreibungstechnik für den locus amoenus. Die Wirklichkeit

9 Cf. Ernst R. Curtius $\left({ }^{2} 1954\right.$ [1948]: 202 [Kap. $10 \$ 6$ »Die Ideallandschaft«], ferner 166-167 [Kap. 8 $\S 4 »$ Gerichts-, Staats- und Lobrede in der mittelalerlichen Poesie«], 168ss. [Kap. $8 \S 5 »$ Unsagbarkeitstopoi«], 171 ss. [Kap. $8 \S 6 »$ Überbietung (als Sonderform des Vergleichs)«]). 
brasilianischer Natur liegt jenseits dieser immer und überall passenden künstlichen bzw. von der Wirklichkeit abstrahierenden Verortung. Der Lustort ist ebenso gültig für die poetisch erfundene Landschaft, in der die bukolische Dichtung spielt, wie für den politisch-oratorischen Lobpreis eines Isidor von Sevilla auf Hispania:

Die verschwenderische Natur hat dich mit allen Arten von Früchten üppig ge-
segnet: Du bist reich an Getier, voller Kraft und heiter mit Saatfeldern. Du stehst
blühend in deinen Ländereien, dichtbelaubt in deinen Bergen, überreich an Fisch
in deinen Gewässern. In der ganzen Welt gibt es kein ähnliches Klima: dich rös-
tet nicht die Glut der Sommersonne, noch lässt dich die Strenge des Winters er-
starren. Eine lauwarme Luft umgibt dich, und sanfte Winde streicheln dich. Was
es auch immer an Anmut auf den Feldern, an kostbaren Metallen und an nütz-
lichen und schönen Tieren gibt, du bringst es hervor.

Auch bei Vespucci liegt die Natur der Neuen Welt als Überschuss hinter den Bildern der Sprache. Sie wird anvisiert und mit Vergleichen vermessen bzw. erst fassbar durch Überbietungsformeln mit der Verstärkung (»molto«) oder im Superlativ und Elativ, oder durch formelhafte Ausdrücke des Staunens, der Verwunderung beziehungsweise, negativ bewertet, des Unglaublichen, ferner durch Topoi der Unsagbarkeit, zu der sowohl die Unzählbarkeit der Tier- und Pflanzenarten als auch die wiederholt eingestreute Versicherung des Autors gehört, er bringe nur Weniges von dem Vielen, das er tatsächlich gesehen habe und von dem er berichten könnte. Das Stilideal der Kürze wird gerade für den Brief (»breve«) empfohlen, doch andererseits steigert die Figur der praeteritio, des Verschweigens und der Auslassung, die Spannung und fordert die Phantasie heraus:

Questa terra è molto amena e piena d'infiniti alberi verdi e molto grandi, e mai non perdono foglie, e tutto hanno odori soavissimi e aromatici, e producono infinitissime frutte, e molte d'esse buone al gusto e salutifere al corpo. E campi producono molte erbe, fiori e radice molto soave e buone [...]. (275)

[Dieses Land ist sehr anmutig, es ist von zahllosen grünen und sehr hohen Bäumen bewachsen, die ihr Laub nie abwerfen, einen sehr süßen Wohlgeruch verbreiten und unendlich viele Früchte hervorbringen, von denen viele wohlschmeckend und gesund sind. Die Felder bringen viele Kräuter und Blumen und Wurzeln hervor, die sehr sü $\beta$ und wohlschmeckend sind.]

10 Concepción Fernández Chicarro (1948). 
Bezeichnend für das rhetorische Beschreibungsverfahren sind Fragenreihen: »Wie sollen wir die zahllosen Vögel schildern, ihre verschiedenen Gefieder, Farben, Gesänge, die Vielfalt und Schönheit der Arten?« (275), oder die bereits erwähnten Verschweigefloskeln: »Trapasso molte cose degne di memoria per non esser più prolisso « (243); »Non voglio alargarmi in questo, perché dubito non sarei creduto«; »chi potrebbe racontare [...]« (243). Bei der Aufzählung von exotischen Tierarten - viele von »gewaltigen Körpermaßen« - kommen sogar Löwen, Hirsche, Hasen und Kaninchen vor, die Vespucci kaum in den von ihm erkundeten Gegenden Brasiliens zu Gesicht bekommen haben dürfte, sie gehören jedoch zum festen Beispielinventar und werden als alte Fruchtbarkeitssymbole formelhaft aufgerufen. Lediglich die Feliden (Raubkatzen) - also wohl zum Beispiel Jaguare sind nicht »so wie « in Spanien, sondern nur in den Antipoden heimisch. Neben der kategorialen Feststellung der Gleichartigkeit (»conformità « [229]) und der durch Vergleich mit dem Bekannten ermittelten Ähnlichkeit wird die Andersartigkeit per viam negationis ausgesagt. Als Gegensatz zu den vielen wildlebenden Tieren (Wildschweine, Böcke, Hirsche, Luchse, Affen, Meerkatzen usw.) stellt Vespucci bündig fest: »Aber Haustiere sahen wir keine« und erfasst damit kulturtypologisch zutreffend den Stand von Jägern und Sammlern in einem der Natur überlassenen Raum, den viele riesenhafte »Waldtiere « (entsprechend silva, silvaticus $>$ salvaje, wild) bevölkern. Im 18. Jahrhundert wird bekanntlich gerade umgekehrt die angebliche Kleinwüchsigkeit der amerikanischen Spezies im Vergleich zu asiatischen und afrikanischen >Großtier〈Arten von Buffon bis Hegel als natürliches Indiz dafür gewertet, dass der amerikanische Kontinent jung, Mundus novus, in der Entwicklungsgeschichte nicht ausgewachsen und gereift und daher auch ohne (Natur-)Geschichte sei: Amerika liege noch im Werden. Im 19. und 20. Jahrhundert erscheint Brasilien als »Land der Zukunft« (Stefan Zweig).

Vespuccis Beschreibungen der Natur gipfeln in zwei überraschenden Pointen, die den Grad der Andersartigkeit hervorheben. Die Darstellung des locus amoenus erreicht den Höhepunkt nach der Beschreibung des milden Klimas und der üppigen Vegetation, bevor die Aufzählung von Tierarten beginnt. Der »süße Duft von Kräutern und Blumen« und der »Geschmack von Früchten und Wurzeln« ruft Erstaunen und Verwunderung hervor. Vespucci schreibt: »Infra me pensavo esser presso al Paradiso terestro« (275). Die Erwähnung des sagenhaften, glücklichen Ortes»Irdi- 
sches Paradies« gehört zum festen Bestandteil von Entdeckerberichten. Die amoenitatis einer friedlichen Gartenlandschaft erscheinen bereits in der biblischen Paradiesvorstellung. Es fällt jedoch auf, dass Vespucci mit einer dreifachen Einschränkung und nicht theologisch auf den Paradiesort anspielt: »Infra me pensavo esser presso al Paradiso terestro, infra questi alimenti arei creduto esser circa a d'esso«, also:

1. »ich dachte bei mir«,

2. "presso al Paradiso« (nahe bei, nicht schon mitten im Paradies) zu sein;

3. mit der dem Briefadressaten in direkter Anrede in den Mund gelegten, scherzhaft vereinnahmenden Vermutung: $» D u$ wirst inmitten dieser Köstlichkeiten auch geglaubt haben (»creduto«), unweit (»circa«) vom Paradies zu sein.«

Die Erwähnung des Inbegriffs eines locus amoenus erfolgt mit listiger Unterscheidung von >denken Möglichkeit, die aber zugleich die skeptische Reserve des gebildeten Kaufherrn gegenüber dergleichen mythischen Wahrnehmungsmustern und den forcierten Versuchen der Lokalisierung des Irdischen Paradieses zum Ausdruck bringt. Dieses >neue Land ‘ ist anders als die Alte Welt, aber trotz der Ursprünglichkeit seiner Natur dieser auch nicht völlig entrückt. Die folgende Beschreibung der »vernünftigen Wesen« und ihrer Lebensweise lässt keineswegs einen paradiesischen Zustand der Menschen >dort erkennen. Sie werden jedoch auch nicht mehr als Fabelwesen oder Monstren aus der Tradition mythischer Welterklärung des Fremden dargestellt. Eine ironische Spitze beschließt die Aufzählung der »vielen anderen Tiere« und die Fragereihen: »Credo che di tante sorte non entrasse nel'arca di Noè « (275276). Gewiss keine respektlose bibelkritische Anspielung auf die im Schöpfungsbericht der Genesis geschilderte Errettung der Tiere aus der Sintflut auf einem kastenförmigen Schiff - und auf Schiffe verstand sich Vespucci nur zu gut, um zu ermessen, welche Fassungskraft sie haben können -; die Erwähnung schränkt einerseits die bekannte biblische Bildvorstellung durch Erfahrung und Wissenschaft ein, erzielt andererseits erneut den rhetorischen Kunsteffekt des überbietenden, paradoxen Vergleichs mit Hilfe des Unsagbarkeitstopos (»unzählig viele Arten«). Zwischen der vermeintlichen Nähe des Irdischen Paradieses und der überbordenden Artenfülle in der Arche steht wieder die Paralipse: »Non voglio alargarmi in 
questo, perché dubito non sarei creduto« (275). Dem Leser wird die leise Ironie dieser Art von Bekräftigung der Glaubwürdigkeit sofort offenkundig. Im Gegensatz zu der im Schema der alten Länderlobtopik gehaltenen descriptio der Natur, die Vespucci in Amerika wahrnahm, wird nun im dritten und längsten Hauptstück über die »vernünftigen Wesen« (»animali razionali«) gehandelt. Die Menschenwelt erscheint im Unterschied zur Natur befremdlich, widersprüchlich, verwirrend, sie ist ganz anders beschaffen als >wir es von >unserem Europa (her kennen. Vespucci stellt hier den Gegensatz von civilización y barbarie fest und bedient sich durchgängig der demonstratio ex negativo, wobei er wiederum zu deren Bekräftigung darauf verweist, er habe siebenundzwanzig Tage lang mit Eingeborenen zusammengelebt, um deren Lebensweise (»mores«) kennenzulernen und zu verstehen (»intendere« [276]). Erfahrung sei besser als jede Theorie, schreibt er an anderer Stelle (234). Als positiv steht zumindest fest, es sind Menschen, »vernünftige Wesen «, von heller Hautfarbe - dadurch also ebenfalls nicht völlig fremdartig - und, was einem Renaissanceitaliener besonders ins Auge sticht: Sie sind wohlgestaltet, von »bella statura«, »gentil disposizione« und ebenmäßigen (235) Gliedmaßen. Damit wird der künstlerische und erotische Schönheitskanon des Italieners uneingeschränkt auf die Neue Welt übertragen. Es sind weder Riesen, noch abartige, körperlich missgestaltete, schreckenerregende Ungeheuer, wie die nach volkstümlicher Vorstellung in entfernten Weltgegenden und in Wäldern hausenden Wilden oder Barbaren eigentlich aussehen müssten, sondern »gente di buono sforzo« (235). Aber die moralisch begründete Verschweigeformel »se tutto s'avessi di contare di quanta poca vergogna tengono, sarebbe entrare in cosa disonesta e miglior è tacerla« (236) eröffnet den sexuellen Phantasien jeglichen Freiraum. Sie sind schön in ihrer kreatürlichen Nacktheit und empfinden darüber nicht einmal Scham. Dies ist ein verwirrender Tabubruch. Denn Nacktheit gilt im Abendland innerhalb der öffentlichen sozialen Verhaltensordnung aus biblisch-moraltheologischem Verständnis heraus als verwerflich, als Zeichen der Erniedrigung (etwa bei Job) und Sündenmal (Adam und Eva). Sie ist insbesondere der Todsünde der luxuria zugeordnet. Vespucci erscheinen die nackten Leiber der amerikanischen Ureinwohner schön im Zustand der Natur (»secondo natura«), in Übereinstimmung mit der vorausgehenden Beschreibung der anmutigen Sinnlichkeit der Naturempfindungen. Der wohlgeformte Leib fasziniert Vespucci, alle anderen zivilisatorischen Merkmale sind dagegen eindeutig negativ be- 
setzt. Entscheidend ist hier das, was fehlt: Nicht das Ähnliche oder Gemeinsame, sondern das ganz Andere wird herausgestrichen. Der Florentiner Stadtbürger legt seiner Beschreibung das Modell der idealen Respubli$c a$, die platonische Staatsauffassung der Polis, zugrunde, die als Maßstab für die Florentiner Verfassung diente. Vespucci fragt in asyndetischer Aufreihung, die eine hämmernde Wirkung hat, die klassischen Merkmale zivilisierten Zusammenlebens ab und trifft jeweils kategorisch verneinte Aussagen: »Non sono«, »non tengono«. Sie haben keine Gesetze und keine Religion. Sie besitzen keine Vorstellung von der Unsterblichkeit der Seele, kennen weder persönliches Eigentum noch Geld, haben keine territorialen Grenzen $^{11}$, keinen König, kein Eisen noch andere Metalle, keine Krankheiten, aber - und das ist der Gipfel befremdlicher Andersartigkeit - sie essen Menschenfleisch, ein weiterer schwerwiegender Tabubruch. Das Bild des

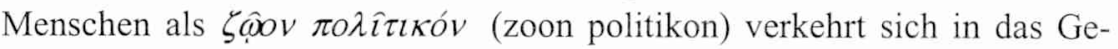
genteil, das Teilhabe am Eigenen oder Eingliederung ausschließt. Der naturbelassene Rohzustand vermag keine anderen als bloß natürliche Verhaltensweisen hervorzubringen. Vespucci erwähnt mit keinem Wort die christliche Mission als Maßnahme zur Gesittung dieser sogenannten primitiven Völkerschaften, denen vermeintlich hierarchische Gliederung, Gesetze und Rechtssprechung, Machtgefüge und Statusdifferenzierung sowie religiöse Kultgemeinschaft als Grundlage und Gewähr für den Bestand von Ordnung und Sittlichkeit fehlen. Obwohl Vespucci trotz der Verständigungsschwierigkeiten, Missverständnisse und fehlenden Begrifflichkeit eine Reihe durchaus signifikanter ethnologischer Befunde festhält - etwa über den rassischen Phänotyp, die >Architektur` der Gemeinschaftshäuser, über Nahrungsmittel, Kalender und Zahlensystem, Rituale und Körperschmuck, der allerdings als ein für den italienischen Geschmack roher, widernatürlicher Brauch abgelehnt wird -, denkt er im Modell des zivilisatorischen Prozesses, dem die antik-christliche Vorstellung der Civitas als Norm zugrunde liegt. Vespucci ist nicht imstande, die Vielfalt und Relativität der forme de vivre anderer Völker anzuerkennen und von den eigenen politischen Erfahrungen und sittlichen Verhaltensregeln abzusehen. Der Vergleich zwischen dem natürlichen Urzustand und dem hochentwickelten Gemeinwesen kann nur Abwertung und Ablehnung bedeuten. Es ist daher schwer nachzuvoll-

11 »Termini« sind nicht »Begriffe«, wie bei Eberhard Schmitt (1984: 177) übersetzt; auf einem Lesefehler beruht auch die Übersetzung »Freundschaft« statt »giustizia« = Justiz! 
ziehen, wie Anneliese Menninger zu dem Urteil gelangen konnte, dass Vespucci die Integration des Fremden in die europäische Vorstellungswelt viel intensiver und umsichtiger gelungen sei als Kolumbus. ${ }^{12}$ Seine Aufmerksamkeit erregen gerade die Abweichungen von europäischen Tabuund Wertvorstellungen (zum Beispiel in Kleidung, Schmuck, Schamempfinden, Sexualverhalten, Körperkraft, Lebensalter, Geburt und Tod). Ausgerechnet bei der Charakterisierung des kriegerischen Wesens der Bewohner, die nackt und ohne Schutzpanzer oder Kampfschild, sowie ohne Taktik und Anführer, aber mit erbarmungsloser Grausamkeit übereinander herfallen - was einen Italiener verwundern muss, der seine Condottieri vor Augen hat -, flicht Vespucci eine poetische, metaphorische Petrarca-Reminiszenz in den Brief ein (278) und bezeichnet »Pfeil und Bogen « der geschickten Schützen als »commessi al vento«. Verwundert fragt er sich mit dem Blick auf die eigene abendländische Geschichts-, Kriegs- und Gewalterfahrung, warum sie eigentlich einander bekriegen, wo sie doch keine Erbschaft, keine Stände- und Rangunterschiede kennen, die allbekannten Ursachen für Streit, Raub und Krieg. Das Prinzip der blutigen Rache und die Demonstration brutaler Kraft treiben sie mit der Gewalt der Instinkte zu grausamen Orgien. Die Schönheit des Leibes auf der positiv besetzten Seite und bestialische Wildheit auf der negativen Seite kennzeichnen die paradox gespannte Differenz zwischen »nostra Europa « und die fehlende »conformità co' nostri di questa parte«. In einem Anflug von Zivilisationskritik am eigenen Zustand vermerkt Vespucci allerdings auch, dass diese Anderen keine unnötigen Bedürfnisse hätten, keinen Luxus kennen und daher auch nicht von Lastern wie »codizia« (ein Hispanismus, < cupiditia, für Habsucht, Raffgier [275]), Geiz, Erbstreitigkeiten, ja selbst von physischen Leiden und Krankheiten geplagt werden, so dass sie steinalt werden können. Den spektakulären und dramatischen Höhepunkt des ethnologischen Briefabschnitts, den Gipfel der Abartigkeit, bildet die Erwähnung des anthropophagischen Tötungsrituals mit einem abwertenden Wortspiel »sagrificio / malifizio« (Opferhandlung / Schandtat [279]). Die Menschenfresserei wird alsbald mit Holzschnitten zu den unter Zuschreibung an Vespucci verbreiteten Drucken und Flugblättern ebenso drastisch wie sensationslüstern ausgeschlachtet. Vespucci sah »zum Räuchern aufgehängtes Menschenfleisch«, löste selbst zehn arme Opfer aus - wo es doch keine Geldwert-

12 Anneliese Menninger (1995: 52). 
vorstellungen gab - und schenkte der Aussage eines Mannes Glauben, der sich damit brüstete, schon »über zweihundert Menschen« verspeist zu haben. »Ich glaube das, und damit genug«, lautet die apodiktische Bekräftigung, obwohl Vespucci an anderer Stelle bekundet, er gehöre zu denen, die es lieber mit dem Hl. Thomas, dem Ungläubigen unter den Jüngern Jesu, halten und langsam im Glauben an das seien, was die Menschen landauf und -ab erzählen. Wie schon der vorhergehende Abschnitt, so endet auch dieser mit einer schelmischen Schlussbemerkung: Wo Menschen, die sich wie Adam und Eva im Paradies ihrer Nacktheit nicht schämten, nicht unter Krankheiten litten und (in kühner Hochrechnung) 130 und mehr Jahre alt würden, ja nicht einmal eines natürlichen Todes stürben, es sei denn »von der Hand eines anderen« oder eben »aus eigener Schuld«, da hätten Ärzte kaum etwas zu vermelden. Dieser bei den Humanisten beliebte Ausfall gegen die Mediziner nimmt die vorausgehenden Aussagen mit Ironie wieder zurück. Zum anderen zielt die Feststellung sowohl auf das Alter des Methusalem, der vor der Sintflut 969 Jahre erreicht habe, als auch auf das überbotene Höchstalter biblischer Lebenserwartung von achtzig Jahren. Wie sollten die Menschen der Neuen Welt denn an die Unsterblichkeit glauben, wenn sie im Grunde gar nicht dem Gesetz des Todes unterworfen sind? Oder erlag Vespucci doch der verführerischen Mythe vom ewigen Jungbrunnen?

Vespucci beschreibt kein Schlaraffenland, seine Schreiben an den gebildeten und mächtigen Mediceer sind ein rhetorisches Kunststück im Umgang mit der inventio und dispositio, mit den capita rerum, auf die er sogar am Schluss augenzwinkernd mit einer abbreviatio (»lassen wir vieles Erwähnenswertes aus, um nicht weitschweifig - prolixus - zu werden « [siehe auch 244]) zurückblickt, nicht ohne allerdings erneut diskret auf seinen demnächst »erscheinenden « ausführlichen Traktat hinzuweisen, den er mit gespielter Bescheidenheit »operetta« (274) (opusculum) nennt. ${ }^{13}$ Wenig glaubwürdig wirkt auch die Beteuerung, dass die im Auftrag der portugiesischen Krone durchgeführte Expedition einzig und allein wissenschaftlicher Erkenntnis und nicht der Suche nach materiellen Reichtümern gedient habe (279). Galt curiositas im Mittelalter als schwere Sünde, wird sie nun als zweckfreies Forschungsinteresse, als Wissensdrang aufgewertet. Ein anderes für den Menschen der Renaissance mit dem Buch und dem Schreiben

13 Im sogenannten Soderini-Brief lautet die Formel »scriuere un zibaldone«. 
eng verbundenes Motiv hatte Vespucci bereits zu Anfang seines Briefes nicht ohne eitles Selbstbewusstsein angesprochen: die fama. Er rühmt sich, ein Viertel der Erde umsegelt zu haben. Lukrative Gesichtspunkte werden gerade durch doppelte Negierungen hervorgehoben. Angeblich finde sich nichts von Nutzen in jener Neuen Welt, und doch preist Vespucci die wunderbare Beschaffenheit des Landes, die Fruchtbarkeit seiner Menschen mit so begeisterten Worten, dass die Aussage über die Nutzlosigkeit nur als Untertreibung zu deuten ist:

Wir fanden [...] so viele Güter, dass sie die heute auf dem Meer fahrenden Schiffe füllen würden, und alle ohne die geringsten Kosten! (280)

Das Prinzip kolonialer Wirtschaft, der Handel mit billigen Kolonialwaren, wird damit vorgezeichnet. Die Bemerkung spekuliert geradezu mit Luxusbedürfnissen und Warenwert, zumal nicht unerwähnt bleibt, wie wenig die Bewohner des Landes Gold, Silber und andere Kostbarkeiten schätzten:

Die Menschen dieses Landes sprechen von Gold und anderen Metallen oder von den wunderbarsten Gewürzen, aber ich gehöre zu denen, die mit dem Hl. Thomas langsam sind im Glauben; die Zeit wird es lehren.

Gespielte, kokette Skepsis des Gelehrten gegenüber den in der Zeit beliebten Vermutungen über die Missionsreisen des Apostels nach Indien oder dissimulatio mit der Absicht, auf verborgene, unermessliche Schätze anzuspielen? Es bleibt der Widerspruch zu der an anderer Stelle gemachten Beobachtung, Eisen und andere Metalle seien unbekannt. Oder könnte es ein rhetorisch geschickt ausgelegter Köder sein, um unter Verbrämung der Dorado-Suche die Neugier zu weiteren Untersuchungen anzustacheln? Vespucci hält sich jedenfalls für künftige Unternehmungen als Experte bereit. Der Brief richtet sich in seiner rhetorischen Strategie auf den fürstlichen Gönner, dessen Wohlwollen Vespucci zu gewinnen oder zu bewahren versucht, ohne die durch seine Aufgabe im Dienst des portugiesischen Königs gebotene Vertraulichkeit zu verletzen. Dieser habe nämlich seine Aufzeichnungen als Geheimsache zunächst persönlich in Gewahrsam genommen.

Die unerhörte Ausweitung des bislang bekannten Raumes der Tierra firme und der Naturkenntnis über das antike Autoritätswissen hinaus wird in ein >Hier und >Dort<, in eine »terra nuova« (der Ausdruck Mundus Novus ist bei Vespucci nicht belegt und geht vielleicht auf Petrus Martyr de Anghiera zurück) und in »nostra Europa « (268) eingeteilt. Differenz und Alterität, Ähnlichkeit und Identität kommen in einem komplizierten Reprä- 
sentationssystem an Fallbeispielen generisch und zugleich aus kritischer oder ironischer Distanz betrachtet zum Ausdruck. Die Neue Welt wird im vergewissernden Rückgriff auf die Alte erfasst, gespiegelt und aufgehoben.

\section{Bibliographie}

\section{Literarische Werke und andere Quellen}

Meyn, Matthias (1984): »Pedro Álvares Cabral nimmt Brasilien für Portugal in Besitz (April 1500)«, in: Schmitt, Eberhard (Hrsg.): Die großen Entdeckungen. Dokumente zur Geschichte der europäischen Expansion, München: Beck, Bd. 2, pp. 171173.

Pozzi, Mario (Hrsg.) (1991): Scopritori e viaggiatori del Cinquecento e del Seicento, Mailand / Neapel: Ricciardi, Bd. 1.

Schmitt, Eberhard / Milan, Carlo (1984): »Amerigo Vespucci berichtet Lorenzo di Pier Francesco de' Medici über die Bewohner der Neuen Welt (1502)«, in: Schmitt, Eberhard (Hrsg.): Die großen Entdeckungen. Dokumente zur Geschichte der europäischen Expansion, München: Beck, Bd. 2, pp. 174-181.

Vespucci, Amerigo (1985): Lettere di viaggio, hrsg. v. Luciano Formisano, Mailand.

Vespucci, Amerigo (1986): Cartas de viaje, hrsg. v. Luciano Formisano, Madrid: Alianza.

\section{Forschungsliteratur}

Bitterli, Urs (1976): Die »Wilden« und die "Zivilisierten«. Grundzüge einer Geistesund Kulturgeschichte der europäisch-überseeischen Begegnung, München: Beck.

Caraccioli Aricò, A. (Hrsg.) (1990): L'impatto della scoperta dell'America nella cultura veneziana, Rom: Bulzoni.

Cardini, Franco (1991): »I Fiorentini e l'espansione europea«, Mare Liberum 2, pp. 31 36.

Colin, Susi (1988): Das Bild des Indianers im 16. Jahrhundert, Idstein: Schulz-Kirchner.

Criado de Val, Manuel (Hrsg.) (1989): Literatura hispánica. Reyes Católicos y Descubrimiento, Barcelona: PPU.

Curtius, Ernst R. ( ${ }^{2} 1954$ [1948]): Europäische Literatur und lateinisches Mittelalter, Bern: Francke.

D'Arienzo, Luisa (1991): »L'influenza culturale italiana alla corte portoghese nell'età delle scoperte«, Mare Liberum 2, pp. 107-120.

Demandt, Alexander $\left({ }^{2} 1993\right.$ [1993]): Der Idealstaat. Die politischen Theorien der Antike, Köln: Böhlau.

Fernández Chicarro, Concepción (1948): Laus Hispaniae, Madrid: Aldus.

Frübis, Hildegard (1995): Die Wirklichkeit des Fremden. Die Darstellung der Neuen Welt im 16. Jahrhundert, Berlin: Reimer.

Gerbi, Antonello (1976): »The earliest accounts on the New World«, in: Chiappelli, Fredi (Hrsg.): First Images of America. The Impact of the New World on the Old, 
Berkeley: UP, Bd. 1, pp. 37-43.

Gerbi, Antonello (1978): La naturaleza de las Indias Nuevas. De Cristóbal Colón a Gonzalo Fernández de Oviedo, Mexiko: Fondo de Cultura Económica.

Gewecke, Frauke (1992): Wie die neue Welt in die alte kam, München: Klett-Cotta.

Greenblatt, Stephen (1994 [1991, Oxford]): Wunderbare Besitztümer. Die Erfindung des Fremden: Reisende und Entdecker, Berlin: Wagenbach.

Guedes, Max Justo / Lombardi, Gerald (Hrsg.) (1990): Portugal Brazil. The Age of Atlantic Discoveries, Lissabon: Bertrand.

Hirsch, Rudolf (1976): »Printed reports on the early discoveries and their reception«, in: Chiappelli, Fredi (Hrsg.): First Images of America. The Impact of the New World on the Old, Berkeley: UP, Bd. 2, pp. 537-562.

Hulme, Peter (1986): Colonial Encounters. Europe and the Native Caribbean 14921797, London / New York.

Keck, Annette / Kording, Inka / Prochaska, Anja (Hrsg.) (1999): Verschlungene Grenzen. Anthropophagie in Literatur und Kulturwissenschaften, Tübingen: Narr.

Kenny, Neil (1998): „Curiosity « in Early Modern Europe, Wiesbaden: Harrassowitz.

Laubenberger, Franz (1982): »The naming of America«, Sixteenth Century Journal 13, pp. 91-113.

Lawrance, Jeremy N. H. (1988): »Nuevos lectores y nuevos géneros: apuntes y observaciones sobre la epistolografía en el primer Renacimiento español«, in: García de la Concha, Víctor (Hrsg.): Literatura en la época del Emperador, Salamanca: Universidad, pp. 81-99.

Leite de Faria, Francisco (1990): »Literary Echoes of the Portuguese Discoveries«, in: Guedes, Max Justo / Lombardi, Gerald (Hrsg.): Portugal Brazil. The Age of Atlantic Discoveries, Lissabon: Bertrand, pp. 235-257.

Luzzana Caraci, Ilaria (1995): "Alle origini della geografia d'America. Le prime edizioni di Mundus Novus«, Geo. Rivista geografica italiana 102, pp. 559-583.

Matos, Luís de (1991): L'expansion portugaise dans la littérature latine de la Renaissance, Lissabon: Fundação Calouste Gulbenkian.

Matos, Luís de (Hrsg.) (1992): Itinerarium Portugallensium, Lissabon: Fundação Calouste Gulbenkian.

Menninger, Anneliese (1995): Die Macht der Augenzeugen. Neue Welt und KannibalenMythos 1492-1600, Stuttgart: Steiner.

Müller, Wolfgang G. (1994): »Brief«, in: Ueding, Gert (Hrsg.): Historisches Wörterbuch der Rhetorik, Tübingen: Niemeyer, Bd. 2, pp. 60-76.

Neuber, Wolfgang (1991): Fremde Welt im europäischen Horizont. Zur Topik der deutschen Amerika-Reiseberichte der Frühen Neuzeit, Berlin: Schmidt.

Oliveira, Julieta Teixeira Marques de (1997): Fontes documentais de Veneza referentes a Portugal, Lissabon: Imprensa Nacional.

Olschki, Leonardo (1922): Geschichte der neusprachlichen wissenschaftlichen Literatur, Leipzig: Olschki, Bd. 2.

Pagden, Anthony (1996): Das erfundene Amerika. Der Aufbruch des europäischen Denkens in die Neue Welt, München: Diederichs.

Pellicer, Rosa (1993): »El Paraíso en el Nuevo Mundo. De Colón a León Pinelo«, in: de las Nieves Muñiz, María (Hrsg.): Espacio geográfico - Espacio imaginario, Cáceres, pp. 135-147.

Pereira, Moacyr Soares (1984): A navegação de 1501 ao Brasil e Américo Vespúcio, Rio de Janeiro: Asa Artes Gráficas. 
Pochat, Götz (1970): Der Exotismus während des Mittelalters und der Renaissance. Voraussetzungen, Entwicklung und Wandlung eines bildnerischen Vokabulars, Stockholm: Almqvist \& Wiksell.

Pögl, Johannes (1986): Die reiche Fracht des Pedro Álvares Cabral. Seine Indische Fahrt und die Entdeckung Brasiliens, Stuttgart / Wien: Thienemann.

Pregliasco, Marinella (1992): »Ritorno ad Antilia«, in: id.: Antilia. Il viaggio e il Mondo Nuovo (XV-XVII secolo), Turin: Einaudi, pp. 165-185.

Ringbom, Lars-Ivar (1958): Paradisus Terrestris. Myt, Bild och Verklighet, Helsingfors.

Romeo, Rosario (1989): Le scoperte americane nella coscienza italiana del Cinquecento, Rom / Bari: Laterza.

Todorov, Tzvetan (1990): »Amerigo Vespucci«, in: Garin, Eugenio (Hrsg.): Der Mensch der Renaissance, Frankfurt am Main: Campus, pp. 350-356.

Wendt, Astrid (1989): Kannibalismus in Brasilien. Eine Analyse europäischer Reiseberichte und Amerika-Darstellungen für die Zeit zwischen 1500 und 1564, Frankfurt am Main: Lang.

Wolff, Hans (1992): »Martin Waldseemüller. Bedeutendster Kosmograph in einer Epoche des forschenden Umbruchs«, in: id. (Hrsg.): America. Das frühe Bild der Neuen Welt, München: Prestel, pp. 111-126.

Ynduráin, Domingo (1988): »Las cartas en prosa«, in: García de la Concha, Víctor (Hrsg.): Literatura en la época del Emperador, Salamanca: Universidad, pp. 53-79. 